ALEA, Lat. Am. J. Probab. Math. Stat. 13, 465-479 (2016)

DOI: 10.30757/ALEA.v13-19

\title{
Preservation properties of stochastic orderings by transformation to Harris family with different tilt parameters
}

\author{
S. Abbasi, M. H. Alamatsaz and E. Cramer \\ Department of Statistics, \\ University of Isfahan, \\ Isfahan, Iran. \\ Department of Statistics, \\ University of Isfahan, \\ Isfahan, Iran. \\ E-mail address: alamatho@sci.ui.ac.ir \\ URL: http://sci.ui.ac.ir/ alamatho \\ Institute of Statistics, \\ RWTH Aachen University, \\ D-52056 Aachen, Germany. \\ E-mail address: erhard.cramer@rwth-aachen.de \\ URL: http://www.isw.rwth-aachen.de/
}

\begin{abstract}
Harris family of distributions models lifetime of a series system when the number of components is a positive random variable. In this paper, we reveal several stochastic comparisons in the Harris family with different tilt parameters and different baseline distributions with respect to the usual stochastic, shifted stochastic, proportional stochastic and shifted proportional stochastic orderings. Such comparisons are particularly useful in lifetime optimization of reliability systems. We shall also present two bounds for a Harris family survival function conditioned on its tilt parameter which are useful when aging properties are considered. Our results generalize several previous findings in this connection.
\end{abstract}

\section{Introduction}

To cover a wide range of data such as those with a high degree of skewness and kurtosis, Marshall and Olkin (1997) and Aly and Benkherouf (2011) introduced two

Received by the editors November 17, 2015; accepted May 13, 2016.

2010 Mathematics Subject Classification. 60E15, 60E05.

Key words and phrases. Aging, Harris distribution, Marshall-Olkin distribution, proportional stochastic ordering, series system, shifted stochastic ordering, shifted proportional stochastic ordering, usual stochastic ordering.

${ }^{1}$ The first author is grateful to the office of Graduate Studies of the University of Isfahan for their support. 
special families of distributions. In their approaches, they considered a baseline distribution and extended it to a new and more flexible distribution. The resulting classes are called Marshall-Olkin and Harris family of distributions, respectively.

The methods of generating Marshall-Olkin and Harris distributions are basically the same. They proceed as follows: Let $Y_{1}, Y_{2}, \ldots$ be a sequence of independent and identical (iid) random variables (rv) with a common distribution function (df) $F$ and survival function (sf) $\bar{F}=1-F$. Let $X=\min \left\{Y_{1}, Y_{2}, \ldots, Y_{N}\right\}$, where $N$ is a positive integer valued $\mathrm{rv}$ independent of the $Y_{i}$ 's with probability generating function (pgf)

$$
P_{N}(t)=E\left(t^{N}\right)=\sum_{n=0}^{\infty} t^{n} P(N=n), \quad t \in[0,1] .
$$

Then, $X$ can be considered as the lifetime of a series system with iid component lifetimes $Y_{1}, Y_{2}, \ldots, Y_{N}$ and a random number $N$ of components. The sf $\bar{H}$ of $X$ has the representation

so that

$$
\bar{H}(x)=\sum_{n=0}^{\infty}[\bar{F}(x)]^{n} P(N=n)
$$

$$
\bar{H}(x)=P_{N}(\bar{F}(x)) .
$$

Assuming $N$ to be a geometric rv, Marshall and Olkin (1997) introduced the socalled Marshall-Olkin distribution. Aly and Benkherouf (2011) used the Harris pgf

$$
P_{N}(s ; \theta, k)=\left\{\frac{\theta s^{k}}{1-\bar{\theta} s^{k}}\right\}^{1 / k}, \quad k>0, \quad 0<\theta<1, \quad \bar{\theta}=1-\theta,
$$

introduced by Harris (1948), and generated the Harris family of survival functions $\bar{H}$ as

$$
\bar{H}(x ; \theta, k)=\left(\frac{\theta \bar{F}^{k}(x)}{1-\bar{\theta} \bar{F}^{k}(x)}\right)^{1 / k}, \quad k>0, \quad 0<\theta<\infty \quad \bar{\theta}=1-\theta .
$$

The df $F$ in (1.3) is called the baseline df and $\theta$ is called the tilt parameter. It is easy to see that hazard rates corresponding to $F$ and $H(\cdot ; \theta, k)$, namely, $r_{F}=f / \bar{F}$ and $r_{H}(. ; \theta, k)=h(. ; \theta, k) / \bar{H}(. ; \theta, k)$, are related by

$$
r_{H}(x ; \theta, k)=\frac{r_{F}(x)}{1-\bar{\theta} \bar{F}^{k}(x)} ; \quad-\infty<x<\infty, \quad 0<\theta<\infty \quad k>0 .
$$

Clearly, $r_{H}(x ; \theta, k)$ exceeds $r_{F}(x)$ when $0<\theta \leq 1$ and it is smaller than $r_{F}(x)$ when $\theta \geq 1$. They coincide when $\theta=1$. Clearly, for $k=1$, pgf (1.2) reduces to the positive geometric pgf which leads to the Marshall-Olkin distribution.

Recently Batsidis and Lemonte (2015) discussed several results in connection with behavior of the failure rate function for Harris family and discussed certain related stochastic orderings. Al-Jarallah et al. (2014) presented a proportional hazard version of Marshall-Olkin family of distribution as $[\bar{H}(. ; \theta, 1)]^{\gamma}$ and investigated likelihood ratio ordering in this model.

Recall that in many applied situations and, in particular, in lifetime systems we need to compare two or more rv's with each other. Comparing only the means (expectation ordering), variances (dispersion ordering) when the means are equal or any other quantitative measures are not adequate. Indeed, in some cases these measures do not exist at all. Therefore, various more useful types of stochastic 
orderings are employed in terms of their survival functions, hazard rate functions, mean residual functions, and other suitable functions of probability distributions. These methods are much more informative than those based on only few numerical characteristics of the underlying distributions. Comparisons of distributions based on such functions usually establish partial orders among rv's. They are called stochastic orderings which play a great role in statistical inference and applied probability. Frequently, they are applied in contexts of Risk Theory, Reliability, Survival Analysis, Economic and Insurance. For example, recently Eryilmaz and Tutuncu (2015) investigated relative behavior of a coherent system with respect to another coherent system using stochastic orderings and Torrado (2015) investigated stochastic properties of the smallest order statistics from Weibull distributions. In Economics, Zhou et al. (2013) developed a new algorithm using stochastic orderings to reduce the search space of maintenance strategies and to enhance the efficiency of optimization algorithms. Nanda and Das (2012) investigated stochastic orderings in Marshal-Olkin family of distributions. Gui (2013) compared Marshall-Olkin power log-normal distributions using stochastic orderings. Ghitany and Kotz (2007) investigated reliability properties of extended linear failure rate distributions using stochastic orderings. Stochastic comparison of different distributions with their mixtures were the concern of Alamatsaz and Abbasi (2008), Jazi and Alamatsaz (2010) and Jazi et al. (2011). Huang and Da (2012) used stochastic orderings to compare members of Marshall-Olkin family.

Recently, Batsidis and Lemonte (2015) were concerned with the behavior of the failure rate function and some stochastic order relations in the Harris family; namely, they compared $H(\cdot ; \theta, k)$ with $H(\cdot ; 1, k)=F$. In the present paper, these results are extended to the case when the tilt parameters may also be different, i.e., we compare $H_{F_{1}}(\cdot ; \alpha, k)$ and $H_{F_{2}}(\cdot ; \beta, k)$ with respect to various stochastic orderings. In particular, we address the usual stochastic orders, various (reversed) hazard rate orderings as well as likelihood ratio orderings (see, e.g., Shaked and Shanthikumar, 2007) to study the stochastic orderings of the Harris family. On one hand, a Harris family distribution can be used as the distribution of the lifetime of a series system and, on the other hand, it is a function of a tilt parameter. Thus, a proper choice of the tilt parameter can play an important role in optimizing the corresponding system's lifetime. In particular, to choose a better model, here, we shall study whether or not certain stochastic orders between the component lifetimes are preserved by the corresponding systems and, more interestingly, vice versa; which is useful in application when the components are not observable. Our results enfold all findings on stochastic orderings of Huang and Da (2012) as special cases who used stochastic orderings to compare members of the Marshall-Olkin family. Recently, Abbasi and Alamatsaz $(2015+)$ investigated preservation properties of stochastic orderings by transformation to Harris family with the same tilt parameters. In Section 2, we introduce various types of stochastic orderings used in the sequel. In Section 3, we discuss the preservation of certain stochastic orderings of the baseline distribution after a transformation to the Harris family under certain conditions on the tilt parameters. In Section 4, using certain aging concepts, we discuss existence of moments of a Harris family distribution and obtain two simple bounds. Notice that Harris family contains all distributions obtained by Marshall-Olkin's method. 


\section{Stochastic Orderings}

Throughout the manuscript, we assume that $X$ and $Y$ are rv's with df's $F$ and $G$, sf's $\bar{F}$ and $\bar{G}$, probability density functions (pdf) $f$ and $g$, hazard rate functions $r_{F}$ and $r_{G}$, reversed hazard rate functions $\tilde{r}_{F}$ and $\tilde{r}_{G}$, and supports $S_{X}$ and $S_{Y}$, respectively. Further, we denote the left and right endpoints of the supports of a r.v. $X$ by $l_{X}$ and $u_{X}$, respectively. We use the terms increasing in place of non-decreasing and decreasing in place of non-increasing. First, we recall some stochastic orderings and present their relations in Table 2.1. For details, we refer to Nakai (1995), Brown and Shanthikumar (1998), Lillo et al. (2001), Shaked and Shanthikumar (2007), and Jarrahiferiz et al. (2013).

Definition 2.1. Stochastic orderings:

(i) $X$ is smaller than $Y$ in the usual stochastic ordering, denoted by $X \leq_{s t} Y$, if $\bar{F}(x) \leq \bar{G}(x), \forall x \in(-\infty, \infty)$.

(ii) $X$ is smaller than $Y$ in the likelihood ratio ordering, denoted by $X \leq_{l r} Y$, if $\frac{g(x)}{f(x)}$ increases in $x$ in $S_{X} \cup S_{Y}$.

(iii) $X$ is smaller than $Y$ in the hazard rate ordering, denoted by $X \leq_{h r} Y$, if $r_{F}(x) \geq r_{G}(x), \forall x \in(-\infty, \infty)$.

(iv) $X$ is smaller than $Y$ in the reversed hazard rate ordering, denoted by $X \leq_{r h}$ $Y$, if $\tilde{r}_{F}(x) \leqslant \tilde{r}_{G}(x), \forall x \in(-\infty, \infty)$.

Definition 2.2. Shifted stochastic orderings:

(i) $X$ is smaller than $Y$ in the up likelihood ratio ordering, denoted by $X \leq_{l r \uparrow} Y$, if $[X-t \mid X>t] \leq_{l r} Y$, for all $t \geq 0$, or, equivalently, if $\frac{g(x)}{f(t+x)}$ increases in $x \in\left[l_{Y}, u_{X}-t\right]$.

(ii) $X$ is smaller than $Y$ in the down likelihood ratio ordering, denoted by $X \leq_{l r \downarrow}$ $Y$, if $X \leq_{l r}[Y-t \mid Y>t]$, for all $t \geq 0$, or, equivalently, if $\frac{g(t+x)}{f(x)}$ increases in $x \in\left[l_{X}, u_{Y}-t\right]$.

(iii) $X$ is smaller than $Y$ in the up hazard rate ordering (up reversed hazard rate ordering), denoted by $X \leq_{h r \uparrow}\left(\leq_{r h \uparrow}\right) Y$, if for all $t \geq 0,[X-t \mid X>t] \leq_{h r}\left(\leq_{r h}\right) Y$ or, equivalently, if $\frac{\bar{G}(x)}{\bar{F}(t+x)}\left(\frac{G(x)}{F(t+x)}\right)$ increases in $x \in\left(-\infty, u_{Y}\right)$, for all $t \geq 0$.

(iv) $X$ is smaller than $Y$ in the down hazard rate ordering (down reversed hazard rate ordering), denoted by $X \leq_{h r \downarrow}\left(\leq_{r h \downarrow}\right) Y$, if for all $t \geq 0, X \leq_{h r}\left(\leq_{r h}\right)[Y-t \mid$ $Y>t]$ or, equivalently, if $\frac{\bar{G}(t+x)}{\bar{F}(x)}\left(\frac{G(t+x)}{F(x)}\right)$ increases in $x \geq 0$, for all $t \geq 0$.

\section{Definition 2.3. Proportional stochastic orderings:}

Let $X$ and $Y$ be continuous and non-negative rv's. Then, $X$ is smaller than $Y$ in the proportional likelihood ratio ordering (plr) (proportional hazard rate ordering (phr), proportional reversed hazard rate ordering (prh)), denoted by $X \leq_{p l r}\left(\leq_{p h r}\right.$ ,$\left.\leq_{p r h}\right) Y$, if for all $0<\lambda \leq 1, \lambda X \leq_{l r}\left(\leq_{h r}, \leq_{r h}\right) Y$ or, equivalently, if $\frac{g(\lambda x)}{f(x)}$ $\left(\frac{\bar{G}(\lambda x)}{\bar{F}(x)}, \frac{G(\lambda x)}{F(x)}\right)$ increases in $x$ for all $0<\lambda \leq 1$ (for details, see Ramos Romero and Sordo Díaz, 2001 and Belzunce et al., 2002).

Jarrahiferiz et al. (2013) have introduced shifted proportional likelihood ratio ordering (shifted proportional hazard rate ordering) for continuous and non-negative rv's as follows: 


\section{Definition 2.4. Shifted proportional stochastic orderings:}

(i) $X$ is smaller than $Y$ in the up proportional likelihood ratio ordering, denoted by $X \leq_{p l r \uparrow} Y$, if $[X-t \mid X>t] \leq_{p l r} Y$ or, equivalently, $\frac{g(\lambda x)}{f(t+x)}$ is increasing in $x \in\left(l_{X}-t, u_{X}-t\right) \cup\left(\frac{l_{Y}}{\lambda}, \frac{u_{Y}}{\lambda}\right)$, for all $t \geq 0$ and $0<\lambda \leq 1$.

(ii) $X$ is smaller than $Y$ in the down proportional likelihood ratio ordering, denoted by $X \leq_{p l r \downarrow} Y$, if $X \leq_{p l r}[Y-t \mid Y>t]$ or, equivalently, if $\frac{g(\lambda x+t)}{f(x)}$ is increasing in $x \geq 0$ for all $t \geq 0$ and $0<\lambda \leq 1$.

(iii) $X$ is smaller than $Y$ in the up proportional hazard rate (up proportional reversed hazard rate) ordering, denoted by $X \leq_{p h r \uparrow}\left(\leq_{p r h \uparrow}\right) Y$, if $[X-t \mid X>$ $t] \leq_{p h r}\left(\leq_{p r h}\right) Y$ or, equivalently, if $\frac{\bar{G}(\lambda x)}{\bar{F}(t+x)}\left(\frac{G(\lambda x)}{F(x+t)}\right)$ is increasing in $x \in\left(0, \frac{u_{Y}}{\lambda}\right)$, for all $t \geq 0$ and $0<\lambda \leq 1$.

(iv) $X$ is smaller than $Y$ in the down proportional hazard rate (down proportional reversed hazard rate)ordering, denoted by $X \leq_{p h r \downarrow}\left(\leq_{p r h \downarrow}\right) Y$, if $X \leq_{p h r}\left(\leq_{p r h}\right.$ )$[Y-t \mid Y>t]$, or, equivalently, if $\frac{\bar{G}(\lambda x+t)}{\bar{F}(x)}\left(\frac{G(\lambda x+t)}{F(x)}\right)$ is increasing in $x \geq 0$ for all $t \geq 0$ and $0<\lambda \leq 1$.

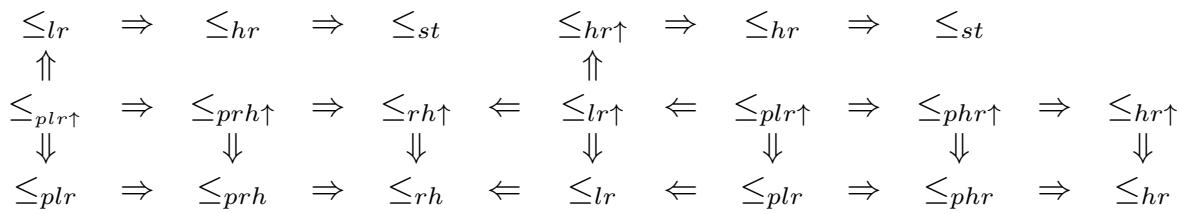

$$
\begin{aligned}
& \Uparrow \Uparrow \Uparrow \quad \Uparrow \quad \Uparrow \quad \Uparrow \quad \text { 介 } \\
& \leq_{p l r \downarrow} \Rightarrow \leq_{p r h \downarrow} \Rightarrow \leq_{r h \downarrow} \Leftarrow \leq_{l r \downarrow} \Leftarrow \leq_{p l r \downarrow} \Rightarrow \leq_{p h r \downarrow} \Rightarrow \leq_{h r \downarrow} \\
& \leq_{s t} \Leftarrow \leq_{r h} \quad \leq_{h r \downarrow}
\end{aligned}
$$

TABLE 2.1. Some useful relations among various types of stochastic orderings

\section{Preservation of stochastic orderings in the Harris family}

Suppose that $Y_{1}$ and $Y_{2}$ are two Harris rv's with df's $H_{1}$ and $H_{2}$ with tilt parameters $\alpha$ and $\beta$, hazard rates $r_{H_{1}}$ and $r_{H_{2}}$ and baseline rv's $X_{1}$ and $X_{2}$ with df's $F_{1}$ and $F_{2}$, respectively. Then, for any $x, \alpha>0, \beta>0$ and $k>0$, pdf's, df's and hazard rate functions associated with $\bar{H}(x ; ., k)$ in $(1.3)$ are given by

$$
\begin{gathered}
h_{1}(x ; \alpha, k)=\frac{\alpha^{\frac{1}{k}} f_{1}(x)}{\left(1-\bar{\alpha} \bar{F}_{1}{ }^{k}(x)\right)^{1+\frac{1}{k}}}, \quad h_{2}(x ; \beta, k)=\frac{\beta^{\frac{1}{k}} f_{2}(x)}{\left(1-\bar{\beta} \bar{F}_{2}^{k}(x)\right)^{1+\frac{1}{k}}}, \\
H_{1}(x ; \alpha, k)=1-\left[\frac{\alpha \bar{F}_{1}^{k}(x)}{1-\bar{\alpha} \bar{F}_{1}^{k}(x)}\right]^{\frac{1}{k}}, \quad H_{2}(x ; \beta, k)=1-\left[\frac{\beta \bar{F}_{2}^{k}(x)}{1-\bar{\beta}_{F_{2}}{ }^{k}(x)}\right]^{\frac{1}{k}},
\end{gathered}
$$

and

$$
r_{H_{1}}(x ; \alpha, k)=\frac{r_{F_{1}}(x)}{1-\bar{\alpha} \bar{F}_{1}{ }^{k}(x)}, \quad r_{H_{2}}(x ; \beta, k)=\frac{r_{F_{2}}(x)}{1-\bar{\beta} \bar{F}_{2}{ }^{k}(x)},
$$

respectively. 
In this section, we study preservation properties of several stochastic orderings of the baseline distribution by its corresponding Harris family distribution. Huang and Da (2012) showed that usual stochastic, hazard rate, reversed hazard rate and likelihood ratio orderings are preserved by a transformation to the Marshall-Olkin family, i.e., for $k=1$ in (1.3). In what follows, their results are generalized to the case $k>0$.

\subsection{Stochastic ordering.}

Theorem 3.1. i) Let $\alpha \leq \beta$. If $X_{1} \leq_{s t} X_{2}$, then $Y_{1} \leq_{s t} Y_{2}$.

ii) Let $\beta \leq \alpha$. If $Y_{1} \leq_{s t} Y_{2}$, then $X_{1} \leq_{s t} X_{2}$.

Proof: i) For any $x$ and $k>0$, we should show that, given $\bar{F}_{1}(x) \leq \bar{F}_{2}(x)$ we have $\bar{H}_{1}(x ; \alpha, k) \leq \bar{H}_{2}(x ; \beta, k)$ or, equivalently,

$$
\left(\frac{\alpha \bar{F}_{1}^{k}(x)}{1-\bar{\alpha} \bar{F}_{1}^{k}(x)}\right)^{\frac{1}{k}} \leq\left(\frac{\beta \bar{F}_{2}^{k}(x)}{1-\bar{\beta} \bar{F}_{2}^{k}(x)}\right)^{\frac{1}{k}} \Longleftrightarrow \frac{\alpha \bar{F}_{1}^{k}(x)}{1-\bar{\alpha} \bar{F}_{1}^{k}(x)} / \frac{\beta \bar{F}_{2}^{k}(x)}{1-\bar{\beta} \bar{F}_{2}^{k}(x)} \leq 1 .
$$

Now, we can write the ratio as

$$
\begin{aligned}
\frac{\frac{\alpha \bar{F}_{1}^{k}(x)}{1-\bar{\alpha} \bar{F}_{1}^{k}(x)}}{\frac{\beta \bar{F}_{2}^{k}(x)}{1-\beta \bar{F}_{2}^{k}(x)}} & =\frac{\alpha \bar{F}_{1}^{k}(x)\left(1-\bar{\beta} \bar{F}_{2}^{k}(x)\right)}{\beta \bar{F}_{2}^{k}(x)\left(1-\bar{\alpha} \bar{F}_{1}^{k}(x)\right)} \\
& =\frac{\alpha \bar{F}_{1}^{k}(x)-\alpha \bar{F}_{1}^{k}(x) \bar{F}_{2}^{k}(x)+\alpha \beta \bar{F}_{1}^{k}(x) \bar{F}_{2}^{k}(x)}{\beta \bar{F}_{2}^{k}(x)-\beta \bar{F}_{1}^{k}(x) \bar{F}_{2}^{k}(x)+\alpha \beta \bar{F}_{1}^{k}(x) \bar{F}_{2}^{k}(x)} \\
& =\frac{\alpha \bar{F}_{1}^{k}(x)\left(1-\bar{F}_{2}^{k}(x)\right)+\alpha \beta \bar{F}_{1}^{k}(x) \bar{F}_{2}^{k}(x)}{\beta \bar{F}_{2}^{k}(x)\left(1-\bar{F}_{1}^{k}(x)\right)+\alpha \beta \bar{F}_{1}^{k}(x) \bar{F}_{2}^{k}(x)} .
\end{aligned}
$$

But,

$$
\begin{aligned}
(3.4) \leq 1 & \Longleftrightarrow \quad \alpha \bar{F}_{1}^{k}(x)\left(1-\bar{F}_{2}^{k}(x)\right)+\alpha \beta \bar{F}_{1}^{k}(x) \bar{F}_{2}^{k}(x) \\
& \leq \beta \bar{F}_{2}^{k}(x)\left(1-\bar{F}_{1}^{k}(x)\right)+\alpha \beta \bar{F}_{1}^{k}(x) \bar{F}_{2}^{k}(x) \\
& \Longleftrightarrow \alpha \bar{F}_{1}^{k}(x)\left(1-\bar{F}_{2}^{k}(x)\right) \leq \beta \bar{F}_{2}^{k}(x)\left(1-\bar{F}_{1}^{k}(x)\right) \\
& \Longleftrightarrow \alpha\left(\bar{F}_{1}^{k}(x)-\bar{F}_{2}^{k}(x)\right) \leq(\beta-\alpha) \bar{F}_{2}^{k}(x)\left(1-\bar{F}_{1}^{k}(x)\right) .
\end{aligned}
$$

Clearly, since $X_{1} \leq s t X_{2}$ means that, for any $k>0, \bar{F}_{1}^{k}(x) \leq \bar{F}_{2}^{k}(x)$, the left hand side of Eq (3.5) is non-positive. Now, if $\alpha \leq \beta$, the right hand side is non-negative. Consequently, we have $Y_{1} \leq_{s t} Y_{2}$.

ii) Now, consider (3.5) with $\beta<\alpha$. Then, it follows that, $Y_{1} \leq_{s t} Y_{2}$ implies $X_{1} \leq_{s t} X_{2}$.

The following counterexample shows that the usual stochastic ordering is not preserved by a transformation to the Harris family when $\alpha>\beta$.

Counterexample 3.1. Let $X_{1}$ and $X_{2}$ be two Lomax rv's with sf's $\bar{F}_{1}(x)=$ $(1+0.3 x)^{-4}$ and $\bar{F}_{2}(x)=(1+0.4 x)^{-2}, x \geq 0$, respectively, so that $X_{1} \leq_{s t} X_{2}$. Figure 3.1 illustrates that, e.g., for $\alpha=4, \beta=1.2, k=2$, and some values of $x$, $\bar{H}_{1}(x ; \alpha, k)>\bar{H}_{2}(x ; \beta, k)$, i.e., $Y_{1} \Varangle_{s t} Y_{2}$. 


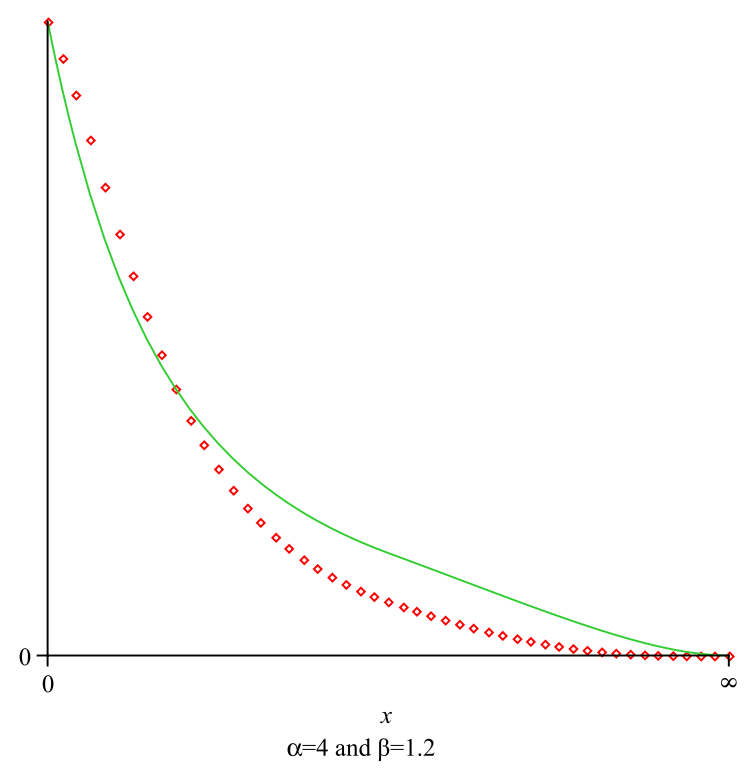

FiguRE 3.1. Illustrating $\bar{H}_{1}(x ; 4,2) \nless \bar{H}_{2}(x ; 1.2,2)$ for the Lomax base distributions.

\subsection{Hazard rate orderings.}

Theorem 3.2. i) Let $\beta \geq \max \{\alpha, 1\}$. If $X_{1} \leq_{p h r \uparrow}\left(\leq_{p h r}, \leq_{h r \uparrow}, \leq_{h r}\right) X_{2}$, then $Y_{1} \leq_{p h r \uparrow}\left(\leq_{p h r}, \leq_{h r \uparrow}, \leq_{h r}\right) Y_{2}$.

ii) Let $\alpha \geq 1$ and $0<\beta \leq 1$. If $Y_{1} \leq_{p h r \uparrow}\left(\leq_{p h r}, \leq_{h r \uparrow}, \leq_{h r}\right) Y_{2}$, then $X_{1} \leq_{p h r \uparrow}$ $\left(\leq_{p h r}, \leq_{h r \uparrow}, \leq_{h r}\right) X_{2}$.

Proof: First note that the usual stochastic order is implied by all the above hazard rate orderings (see Table 2.1) discussed in the theorem. Thus, we have $\bar{F}_{1}^{k}(x) \leq$ $\bar{F}_{2}^{k}(x)$ for any $k>0$.

i) By Definition 2.4 (iii), $X_{1} \leq_{p h r \uparrow} X_{2}$ implies that $r_{F_{1}}(x+t) \geq \lambda r_{F_{2}}(\lambda x) \geq 0$ for any $x$, all $t \geq 0$ and all $0<\lambda \leq 1$. Since $\bar{F}$ is decreasing, we have $\bar{F}_{1}^{k}(x+t) \leq \bar{F}_{1}^{k}(x)$ and $\bar{F}_{2}^{k}(x) \leq \bar{F}_{2}^{k}(\lambda x)$. For $\alpha \geq 1$ and $\beta \geq \alpha$, we get $1-\bar{\alpha} \bar{F}_{1}^{k}(x+t) \leq 1-\bar{\beta} \bar{F}_{2}^{k}(\lambda x)$ or, $\frac{1}{1-\bar{\alpha} F_{1}^{k}(x+t)} \geq \frac{1}{1-\bar{\beta} \bar{F}_{2}^{k}(\lambda x)}$. Hence, by Eq. (3.3) and Definition 2.4 (iii), we have that $Y_{1} \leq_{p h r \uparrow} Y_{2}$.

For $0<\alpha \leq 1$ and $\beta \geq 1$, we have $\bar{\alpha} \geq 0$ and $\bar{\beta} \leq 0$ so that $\bar{\alpha} \bar{F}_{1}^{k}(x+t) \geq \bar{\beta} \bar{F}_{2}^{k}(\lambda x)$ or, $1-\bar{\alpha} \bar{F}_{1}^{k}(x+t) \leq 1-\bar{\beta} \bar{F}_{2}^{k}(\lambda x)$ which yields $\frac{1}{1-\bar{\alpha} \bar{F}_{1}^{k}(x+t)} \geq \frac{1}{1-\bar{\beta} \bar{F}_{2}^{k}(\lambda x)} \geq 0$. Therefore, by Eq. (3.3) and Definition 2.4 (iii), we conclude that $Y_{1} \leq{ }_{p h r \uparrow} Y_{2}$.

ii) By Definition 2.4 (iii), $Y_{1} \leq_{p h r \uparrow} Y_{2}$ implies that $r_{H_{1}}(x+t) \geq \lambda r_{H_{2}}(\lambda x) \geq 0$ for any $x$, all $t \geq 0$ and all $0<\lambda \leq 1$. Thus, by Eq. (3.3),

$$
\frac{r_{F_{1}}(x+t)}{\lambda r_{F_{2}}(\lambda x)} \geq \frac{1-\bar{\alpha} \bar{F}_{1}^{k}(x+t)}{1-\bar{\beta} \bar{F}_{2}^{k}(\lambda x)} \text {. }
$$

Since $\alpha \geq 1$ and $0<\beta \leq 1$, we have $\bar{\alpha} \bar{F}_{1}^{k}(x+t) \leq \bar{\beta} \bar{F}_{2}^{k}(\lambda x)$ or, $1-\bar{\alpha} \bar{F}_{1}^{k}(x+t) \geq$ $1-\bar{\beta} \bar{F}_{2}^{k}(\lambda x)$ which yields that the right side of Inequality (3.6) is greater than or equal to 1 . Thus, by Inequality (3.6), $r_{F_{1}}(x+1) \geq \lambda r_{F_{2}}(\lambda x)$ and thus, $X_{1} \leq_{p h r \uparrow} X_{2}$. 
With proper choices of $t(=0)$ or $\lambda=1$ or both, proofs of the other parts are immediate.

The following counterexample shows that up proportional hazard rate ordering is not preserved by transformation to the Harris family when $\beta \ngtr \max \{\alpha, 1\}$.

Counterexample 3.2. Let $X_{1}$ and $X_{2}$ be two Erlangian distributed rv's with sf's $\bar{F}_{1}(x)=(1+2 x) e^{-2 x}$ and $\bar{F}_{2}(x)=(x+1) e^{-x}$ and hazard rate functions $r_{F_{1}}(x)=\frac{4 x}{1+2 x}$ and $r_{F_{2}}(x)=\frac{x}{x+1}$, for $x>0$, respectively. For any $0<\lambda \leq 1, t \geq 0$ and $x$ we have $r_{F_{1}}(x+t) \geq \lambda r_{F_{2}}(\lambda x)$. Thus, by Definition 2.4 (iii), $X_{1} \leq_{p h r \uparrow}$ $X_{2}$. Figure 3.2 shows that in four different situations where $\beta \ngtr \max \{\alpha, 1\}$, $\bar{H}_{2}(\lambda x ; \beta, k) / \bar{H}_{1}(x+t ; \alpha, k)$ is not increasing in $x$ or, equivalently, for some values of $x, r_{H_{1}}(x+t ; \alpha, k) \ngtr \lambda r_{H_{2}}(\lambda x ; \beta, k)$, i.e., up proportional hazard rate ordering is not preserved by transformation to Harris family when $\beta \ngtr \max \{\alpha, 1\}$.
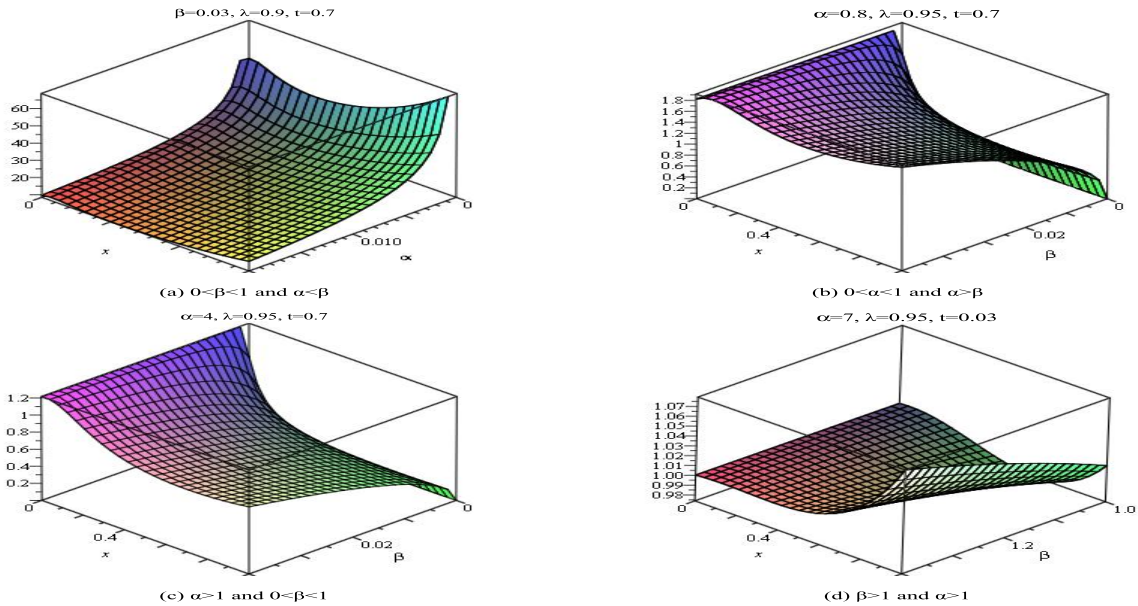

FIGURE 3.2. Showing that $\bar{H}_{2}(\lambda x ; \beta, 2) / \bar{H}_{1}(x+t ; \alpha, 2)$ is not increasing in $x$ when $\beta \leq \max \{\alpha, 1\}$.

Theorem 3.3. i) Let $0<\alpha \leq 1$ and $\beta \geq 1$. If $X_{1} \leq_{p h r \downarrow}\left(\leq_{h r \downarrow}\right) X_{2}$, then $Y_{1} \leq_{p h r \downarrow}$ $\left(\leq_{h r \downarrow}\right) Y_{2}$,

ii) Let $0<\beta \leq 1$ and $\alpha \geq 1$. If $Y_{1} \leq_{p h r \downarrow}\left(\leq_{h r \downarrow}\right) Y_{2}$, then $X_{1} \leq_{p h r \downarrow}\left(\leq_{h r \downarrow}\right) X_{2}$.

Proof: i) By Definition 2.4 (iv), $X_{1} \leq_{p h r \downarrow} X_{2}$ is equivalent to $r_{F_{1}}(x) \geq \lambda r_{F_{2}}(\lambda x+t)$ for any $x, 0<\lambda \leq 1$ and $t \geq 0$. Furthermore, for $0<\alpha \leq 1$ and $\beta \geq 1$, and any $k>0$, we have $\bar{\alpha} \bar{F}_{1}^{k}(x) \geq \bar{\beta} \bar{F}_{2}^{k}(\lambda x+t)$, or $0 \leq 1-\bar{\alpha} \bar{F}_{1}^{k}(x) \leq 1-\bar{\beta} \bar{F}_{2}^{k}(x+t)$ which yields $\frac{1}{1-\bar{\alpha} \bar{F}_{1}^{k}(x)} \geq \frac{1}{1-\bar{\beta} \bar{F}_{2}^{k}(\lambda x+t)} \geq 0$. Thus, by Eq. (3.3) and Definition 2.4 (iv) we conclude that $Y_{1} \leq p h r \downarrow Y_{2}$.

ii) By Definition 2.4 (iv), $Y_{1} \leq_{p h r \downarrow} Y_{2}$ is equivalent to $r_{H_{1}}(x) \geq \lambda r_{H_{2}}(\lambda x+t)$ for any $x, 0<\lambda \leq 1$ and $t \geq 0$. Thus by Eq. (3.3),

$$
\frac{r_{F_{1}}(x)}{\lambda r_{F_{2}}(\lambda x+t)} \geq \frac{1-\bar{\alpha} \bar{F}_{1}^{k}(x)}{1-\bar{\beta} \bar{F}_{2}^{k}(\lambda x+t)} .
$$

Since $\alpha \geq 1$ and $0<\beta \leq 1$ for any $k>0$, we have $\bar{\alpha} \bar{F}_{1}^{k}(x) \leq \bar{\beta} \bar{F}_{2}^{k}(\lambda x+t)$, or $0 \leq 1-\bar{\alpha} \bar{F}_{1}^{k}(x) \geq 1-\bar{\beta} \overline{\bar{F}}_{2}^{k}(x+t)$ which implies that the right side of Inequality 
(3.7) is greater than or equal to 1 and consequently $r_{F_{1}}(x) \geq \lambda r_{F_{2}}(\lambda x+t)$. As required.

With the choice of $\lambda=1$, proofs of down hazard rate orderings are immediate.

The following counterexample shows that down proportional hazard rate ordering is not preserved by transformation to the Harris family when $\alpha$ and $\beta$ do not satisfy the conditions of Theorem 3.3.

Counterexample 3.3. Let $X_{1}$ and $X_{2}$ be two exponential rv's with hazard rates 3 and 2, respectively. For any $x, 0<\lambda \leq 1$ and $t \geq 0$ we have $r_{F_{1}}(x) \geq r_{F_{2}}(\lambda x+t)$. Thus, by Definition 2.4 (iv), we conclude that $X_{1} \leq_{p h r \downarrow} X_{2}$. We see that for some values of $0<\lambda \leq 1, \alpha, \beta$ and $t \geq 0$, such that $\alpha$ and $\beta$ do not satisfy the conditions of Theorem 3.3 (for example: take $\alpha=8, \lambda=0.9, t=0.1$ and $0<\beta<1), \frac{\bar{H}_{2}(\lambda x+t ; \beta, k)}{\bar{H}_{1}(x ; \alpha, k)}$ is not increasing in $x$ or, equivalently, for some values of $x, r_{H_{1}}(x ; \alpha, k) \nsupseteq \lambda r_{H_{2}}(\lambda x+t ; \beta, k)$, i.e., down proportional hazard rate ordering is not preserved by transformation to the Harris family.

\subsection{Likelihood ratio orderings.}

Theorem 3.4. i) Let $0<\alpha \leq 1$ and $\beta \geq 1$. If $X_{1} \leq_{p l r \uparrow}\left(\leq_{p l r}, \leq_{l r \uparrow}, \leq_{l r}\right) X_{2}$, then $Y_{1} \leq_{p l r \uparrow}\left(\leq_{p l r}, \leq_{l r \uparrow}, \leq_{l r}\right) Y_{2}$.

ii) Let $0<\beta \leq 1$ and $\alpha \geq 1$. If $Y_{1} \leq_{p l r \uparrow}\left(\leq_{p l r}, \leq_{l r \uparrow}, \leq_{l r}\right) Y_{2}$, then $X_{1} \leq_{p l r \uparrow}\left(\leq_{p l r}\right.$ $\left., \leq_{l r \uparrow}, \leq_{l r}\right) X_{2}$.

Proof: i) By Definition 2.4 (i), we have to show that, for all $0<\lambda \leq 1, t \geq 0$ and $k>0, \frac{h_{1}(x+t ; \alpha, k)}{h_{2}(\lambda x ; \beta, k)}$ is decreasing in $x$. But, by Eq. (3.1),

$$
\frac{h_{1}(x+t ; \alpha, k)}{h_{2}(\lambda x ; \beta, k)}=\left(\frac{\alpha}{\beta}\right)^{\frac{1}{k}} \cdot \frac{f_{1}(x+t)}{f_{2}(\lambda x)} \cdot\left(\frac{1-\bar{\beta} \bar{F}_{2}^{k}(\lambda x)}{1-\bar{\alpha} \bar{F}_{1}^{k}(x+t)}\right)^{\frac{1}{k}+1},
$$

and $X_{1} \leq_{p l r \uparrow} X_{2}$ implies that, for all $0<\lambda \leq 1$ and $t \geq 0, \frac{f_{1}(x+t)}{f_{2}(\lambda x)}$ is decreasing in $x$. Further, we have

$$
\begin{aligned}
& \frac{d}{d x}\left(\frac{1-\bar{\beta} \bar{F}_{2}^{k}(\lambda x)}{1-\bar{\alpha} \bar{F}_{1}^{k}(x+t)}\right) \\
& =\frac{k \bar{\alpha} \bar{\beta} \bar{F}_{1}^{k-1}(x+t) \bar{F}_{2}^{k-1}(\lambda x)\left[f_{1}(x+t) \bar{F}_{2}(\lambda x)-\lambda f_{2}(\lambda x) \bar{F}_{1}(x+t)\right]}{\left(1-\bar{\alpha} \bar{F}_{1}^{k}(x)\right)^{2}} \\
& +\frac{k\left[\bar{\beta} \lambda f_{2}(\lambda x) \bar{F}_{2}^{k-1}(\lambda x)-\bar{\alpha} f_{1}(x+t) \bar{F}_{1}^{k-1}(x+t)\right]}{\left(1-\bar{\alpha} \bar{F}_{1}^{k}(x)\right)^{2}} .
\end{aligned}
$$

Using that up proportional hazard rate ordering is implied by up proportional likelihood ratio ordering (see Table 2.1), the first term in Eq. (3.8) is non-positive. Moreover, for $0 \leq \alpha \leq 1$ and $\beta \geq 1$, the second term is also non-positive. Thus, since both terms of Eq. (3.8) are non-negative, we have the result.

ii) By Definition 2.4 (i), for all $0<\lambda \leq 1, t \geq 0$ and $k>0, \frac{h_{1}(x+t ; \alpha, k)}{h_{2}(\lambda x ; \beta, k)}$ is decreasing in $x$, i.e., for any $x \leq y, h_{1}(y+t ; \alpha, k) h_{2}(\lambda x ; \beta, k) \leq h_{1}(x+t ; \alpha, k) h_{2}(\lambda y ; \beta, k)$. So, by Eq. (3.1), we have

$$
\left[\frac{1-\bar{\alpha} \bar{F}_{1}^{k}(x+t)}{1-\bar{\alpha} \bar{F}_{1}^{k}(y+t)}\right]^{\frac{k+1}{k}}\left[\frac{1-\bar{\beta} \bar{F}_{2}^{k}(\lambda y)}{1-\bar{\beta} \bar{F}_{2}^{k}(\lambda x)}\right]^{\frac{k+1}{k}} \leq \frac{f_{1}(x+t)}{f_{2}(\lambda x)} \frac{f_{2}(\lambda y)}{f_{1}(y+t)} .
$$


By decreasing property of sf's, we have $\bar{F}_{1}^{k}(y+t) \leq \bar{F}_{1}^{k}(x+t)$. If $\alpha>1$, then $\bar{\alpha} \bar{F}_{1}^{k}(y+t) \geq \bar{\alpha} \bar{F}_{1}^{k}(x+t)$ and $1-\bar{\alpha} \bar{F}_{1}^{k}(y+t) \geq 1-\bar{\alpha} \bar{F}_{1}^{k}(x+t)$. Thus, the first factor in the left side of Inequality (3.9) is greater than or equal to 1. Moreover, if $0<\beta \leq 1$, since sf is decreasing, we have also $1-\bar{\beta} \bar{F}_{2}^{k}(\lambda x) \leq 1-\bar{\beta} \bar{F}_{2}^{k}(\lambda y)$. Thus, the second factor in the left side of Inequality (3.9) is greater than or equal to 1. So, we conclude that the right side of Inequality (3.9) is greater than or equal to 1 and by Definition 2.4 (i) we have the result.

With proper choices of $t(=0)$ and $\lambda=1$ or both, proofs of the other parts are immediate.

Theorem 3.5. i) Let $0<\alpha \leq 1$ and $\beta \geq 1$. If $X_{1} \leq_{p l r \downarrow}\left(\leq_{l r \downarrow}\right) X_{2}$, then $Y_{1} \leq_{p l r \downarrow}$ $\left(\leq_{l r \downarrow}\right) Y_{2}$.

ii) Let $0<\beta \leq 1$ and $\alpha \geq 1$. If $Y_{1} \leq_{p l r \downarrow}\left(\leq_{l r \downarrow}\right) Y_{2}$, then $X_{1} \leq_{p l r \downarrow}\left(\leq_{l r \downarrow}\right) X_{2}$.

Proof: i) By Definition 2.4 (ii), we have to show that for all $t \geq 0,0<\lambda \leq 1$ and $k>0, \frac{h_{1}(x ; \alpha, k)}{h_{2}(\lambda x+t ; \beta, k)}$ is decreasing in $x$. By Eq. (3.1), we have

$$
\frac{h_{1}(x ; \alpha, k)}{h_{2}(\lambda x+t ; \beta, k)}=\left(\frac{\alpha}{\beta}\right)^{\frac{1}{k}} \cdot \frac{f_{1}(x)}{f_{2}(\lambda x+t)} \cdot\left(\frac{1-\bar{\beta} \bar{F}_{2}^{k}(\lambda x+t)}{1-\bar{\alpha} \bar{F}_{1}^{k}(x)}\right)^{\frac{1}{k}+1} .
$$

Thus, since $X_{1} \leq_{p l r \downarrow} X_{2}$ implies that, for all $t \geq 0$ and $0<\lambda \leq 1, \frac{f_{1}(x)}{f_{2}(\lambda x+t)}$ is decreasing in $x$ and both factors of Eq. (3.10) are non-negative, it is sufficient to show that $\left(\frac{1-\bar{\beta} \bar{F}_{2}^{k}(\lambda x+t)}{1-\bar{\alpha} \bar{F}_{1}^{k}(x)}\right)^{\frac{1}{k}+1}$, or $\frac{1-\bar{\beta} \bar{F}_{2}^{k}(\lambda x+t)}{1-\bar{\alpha} \bar{F}_{1}^{k}(x)}$, is decreasing in $x$. But, we have

$$
\begin{aligned}
& \frac{d}{d x}\left(\frac{1-\bar{\beta} \bar{F}_{2}^{k}(\lambda x+t)}{1-\bar{\alpha} \bar{F}_{1}^{k}(x)}\right) \\
& =\frac{k \bar{\alpha} \bar{\beta} \bar{F}_{1}^{k-1}(x) \bar{F}_{2}^{k-1}(\lambda x+t)\left[f_{1}(x) \bar{F}_{2}(x+t)-\lambda f_{2}(\lambda x+t) \bar{F}_{1}(x)\right]}{\left(1-\bar{\alpha} \bar{F}_{1}^{k}(x)\right)^{2}} \\
& +\frac{k\left[\bar{\beta} \lambda f_{2}(\lambda x+t) \bar{F}_{2}^{k-1}(\lambda x+t)-\bar{\alpha} f_{1}(x) \bar{F}_{1}^{k-1}(x)\right]}{\left(1-\bar{\alpha} \bar{F}_{1}^{k}(x)\right)^{2}} .
\end{aligned}
$$

The first term in Eq. (3.11) is non-positive because down proportional hazard rate ordering is implied by down proportional likelihood ratio ordering (see Table 2.1). Moreover, for $0 \leq \alpha \leq 1$ and $\beta \geq 1$, the second term in Eq. (3.11) is also non-positive. Thus, we have proved the result.

ii) By Definition 2.4 (ii), for all $0<\lambda \leq 1, t \geq 0$ and $k>0, \frac{h_{1}(x ; \alpha, k)}{h_{2}(\lambda x+t ; \beta, k)}$ is decreasing in $x$, i.e., for all $x \leq y, h_{1}(y ; \alpha, k) h_{2}(\lambda x+t ; \beta, k) \leq h_{1}(x ; \alpha, k) h_{2}(\lambda y+$ $t ; \beta, k)$. So, by Eq. (3.1), we have

$$
\left[\frac{1-\bar{\alpha} \bar{F}_{1}^{k}(x)}{1-\bar{\alpha} \bar{F}_{1}^{k}(y)}\right]^{\frac{k+1}{k}}\left[\frac{1-\bar{\beta} \bar{F}_{2}^{k}(\lambda y+t)}{1-\bar{\beta} \bar{F}_{2}^{k}(\lambda x+t)}\right]^{\frac{k+1}{k}} \leq \frac{f_{1}(x)}{f_{2}(\lambda x+t)} \frac{f_{2}(\lambda y+t)}{f_{1}(y)}
$$

By the decreasing property of sf, we have $\bar{F}_{1}^{k}(y) \leq \bar{F}_{1}^{k}(x)$. If $\alpha>1$, we have $1-\bar{\alpha} \bar{F}_{1}^{k}(y) \geq 1-\bar{\alpha} \bar{F}_{1}^{k}(x)$. Thus, the first factor in the left side of Inequality (3.12) is greater than or equal to 1 . Moreover, if $0<\beta \leq 1$, since sf is decreasing, we have $1-\bar{\beta} \bar{F}_{2}^{k}(\lambda x+t) \leq 1-\bar{\beta} \bar{F}_{2}^{k}(\lambda y+t)$. Thus, the second factor in the left side of Inequality (3.12) is greater than or equal to 1 . Hence, the right side of Inequality (3.12) is greater than or equal to 1 and by Definition 2.4 (ii) we have the result.

By choosing $\lambda=1$, proofs of the other parts are immediate. 
The following counterexample shows that up proportional likelihood ratio ordering is not preserved by transformation to Harris family when the conditions of Theorem 3.4 on $\alpha$ and $\beta$ are not satisfied.

Counterexample 3.4. Let $X_{1}$ and $X_{2}$ be two Rayleigh rv's with pdf's $f_{1}(x)=$ $\frac{x}{0.01} \exp \left(\frac{-x^{2}}{0.02}\right)$ and $f_{2}(x)=\frac{x}{0.04} \exp \left(\frac{-x^{2}}{0.08}\right)$, respectively. For all $0<\lambda \leq 1$ and $t \geq 0$, $\frac{f_{1}(x+t)}{f_{2}(\lambda x)}$ is decreasing in $x$, thus by Definition $2.4(\mathrm{i}), X_{1} \leq_{p l r \uparrow} X_{2}$. We can show that for some values of $\alpha, \beta, 0<\lambda \leq 1$ and $t \geq 0$ not satisfying the condition in Theorem 3.4 (for example, take $\alpha=5, t=0.01, \lambda=0.9$ and $0<\beta<1$ ), $\frac{h_{1}(x+t ; \alpha, k)}{h_{2}(\lambda x ; \beta, k)}$ is not decreasing in $x$, i.e., up proportional likelihood ratio ordering is not preserved by transformation to the Harris family in this situation.

The following counterexample shows that down proportional likelihood ratio ordering is not preserved by transformation to the Harris family when $\alpha$ and $\beta$ do not satisfy the conditions of Theorem 3.5.

Counterexample 3.5. Let $X_{1}$ and $X_{2}$ be two rv's with pdf's $f_{1}(x)=\frac{1}{2} \exp \left(\frac{x}{2}\right)$ and $f_{2}(x)=\frac{x}{9} \exp \left(-\frac{x}{3}\right)$, respectively. For all $0<\lambda \leq 1$ and $t \geq 0, \frac{f_{1}(x)}{f_{2}(\lambda x+t)}$ is decreasing in $x$, thus by Definition 2.4 (ii), $X_{1} \leq_{p l r \downarrow} X_{2}$. Again we see that for some values of $0<\lambda \leq 1, \alpha, \beta$ and $t \geq 0$ which do not satisfy the condition in Theorem 3.5 (for example, take $\alpha=5, t=5, \lambda=0.9$ and $\beta>1$ ), $\frac{h_{1}(x ; \alpha, k)}{h_{2}(\lambda x+t ; \beta, k)}$ is not decreasing in $x$, i.e., down proportional likelihood ratio ordering is not preserved by transformation to the Harris family.

3.4. Reversed hazard rate orderings. Huang and Da (2012) have investigated the preservation of hazard rate ordering by transformation to Marshall-Olkin family but have remained silent in the reversed hazard rate orderings. In what follows, we complete their study by considering preservation properties of up reversed hazard rate, proportional reversed hazard rate and up proportional reversed hazard rate orderings in Marshall-Olkin family. For the general case of $k>0$, i.e., Harris family, the result remains as a conjecture to be proved.

Theorem 3.6. Let $\alpha<\min \{\beta, 1\}$ and $k=1$. If $X_{1} \leq_{p r h \uparrow}\left(\leq_{p r h}, \leq_{r h \uparrow}\right) X_{2}$, then $Y_{1} \leq_{p r h \uparrow}\left(\leq_{p r h}, \leq_{r h \uparrow}\right) Y_{2}$.

Proof: For any $0<\lambda \leq 1, t \geq 0$ we have

$$
\frac{H_{1}(x+t ; \alpha, 1)}{H_{2}(\lambda x ; \beta, 1)}=\frac{1-\frac{\alpha \bar{F}_{1}(x+t)}{\left(1-\bar{\alpha} \bar{F}_{1}(x+t)\right)}}{1-\frac{\beta \bar{F}_{2}(\lambda x)}{\left(1-\bar{\beta} \bar{F}_{2}(\lambda x)\right)}}=\frac{\frac{\beta}{F_{2}(\lambda x)}+\bar{\beta}}{\frac{\alpha}{F_{1}(x+t)}+\bar{\alpha}} .
$$

By Definition 2.4 (iii), we have to show that, for any $0<\lambda<1, t \geq 0, \frac{H_{1}(x+t ; \alpha, 1)}{H_{2}(\lambda x ; \beta, 1)}$ is decreasing in $x$. Now,

$\frac{d}{d x}\left(\frac{H_{1}(x+t ; \alpha, 1)}{H_{2}(\lambda x ; \beta, 1)}\right)=\frac{\frac{\alpha \beta}{F_{2}(\lambda x) F_{1}(x+t)}\left[\frac{f_{1}(x+t)}{F_{1}(x+t)}-\frac{\lambda f_{2}(\lambda x)}{F_{2}(\lambda x)}\right]+\left[\frac{\alpha \bar{\beta} f_{1}(x+t)}{F_{1}^{2}(x+t)}-\frac{\bar{\alpha} \beta \lambda f_{2}(\lambda x)}{F_{2}^{2}(\lambda x)}\right]}{\left(\frac{\alpha}{F_{1}(x+t)}+\bar{\alpha}\right)^{2}}$.

Thus, since $X_{1} \leq_{p r h \uparrow} X_{2}$ is equivalent to $\frac{F_{1}(x+t)}{F_{2}(\lambda x)}$ being decreasing in $x$ for any $0<\lambda \leq 1$ and $t \geq 0$, the first term in the numerator of Eq. (3.13) is non-positive. Moreover, the stochastic order is implied by up proportional reversed hazard rate ordering (see Table 2.1), so $F_{2}(x) \leq F_{1}(x)$ and, by the monotonicity of df $s$, it follows 
that $F_{2}(\lambda x) \leq F_{1}(x+t)$, for any $0<\lambda \leq 1$ and $t \geq 0$. Thus, if $\alpha<\min \{\beta, 1\}$, the second term in the numerator of Eq. (3.13) is also non-positive. This completes the proof.

With proper choices of $t(=0)$ or $\lambda(=1)$ or both, proofs of the other parts are immediate.

Counterexample 3.6. Let $X_{1}$ and $X_{2}$ be two exponentially distributed rv's with sf's $\bar{F}_{1}(x)=e^{-8 x}$ and $\bar{F}_{2}(x)=e^{-4 x}$ for $x>0$, respectively. In this case, for any $0<\lambda \leq 1, t \geq 0, \frac{f_{1}(x+t)}{f_{2}(\lambda x)}$ is decreasing in $\mathrm{x}$. Thus, by Definition $2.4(\mathrm{i}), X_{1} \leq_{p l r \uparrow} X_{2}$. Since up proportional reversed hazard rate ordering is implied by up proportional likelihood ratio, we conclude that $X_{1} \leq_{p r h \uparrow} X_{2}$. It can be observed that for some values of $\alpha, \beta, 0<\lambda \leq 1$ and $t \geq 0$ not satisfying the condition in Theorem 3.6 (for example, take $\alpha=9, t=0.3, \lambda=0.8$ and $0<\beta<1), H_{2}(\lambda x ; \beta, 1) / H_{1}(x+t ; \alpha, 1)$ is not increasing in $x$, i.e., up proportional reversed hazard rate ordering is not preserved by transformation to Harris family when $\alpha \nless \min \{\beta, 1\}$.

\section{Aging properties}

We should point out that in many practical problems, when using a sample data set, we are able to obtain some life information such as the mean and variance of the life distribution. But the exact value of the reliability function can not be easily obtained. However, it is still helpful to derive some bounds for a reliability function based on the known information. These bounds can tell us the scope of the reliability of products and provide a basis for further improvements. In this section, we obtain two bounds for survival functions conditioned on the tilt random parameter, which are useful in distinguishing the failure probability of a component after a time t.

Proportional failure rate was defined by Singh and Maddala (1976) and was called generalized failure rate by Lariviere and Porteus (2001). The generalized failure rate is defined as $g_{F}(x)=x r_{F}(x)$. A rv $X$ has increasing generalized failure rate (IGFR) property if $g_{F}(x)$ is increasing in $x$ such that $F(x)<1$. IGFR is useful and frequently used in recent pricing, revenue and supply chain management literature. Now using the concept IGFR, we discuss existence of moments of a Harris family distribution.

Theorem 4.1. Let $X$ and $Y$ be two non-negative rv's corresponding to $F($.$) and$ $H(. ; \theta, k)$ in Eq. (1.3) with $\theta \geq 1$, respectively. Suppose that $X$ has the IGFR property and $\lim _{x \rightarrow \infty} g_{F}(x)=l$, where $l$ is possibly infinite. Then, $E\left(Y^{n}\right), n>0$, is finite if, and only if, $l>n$.

Proof: If $\theta \geq 1$, then $1-\bar{\theta} \bar{F}^{k}(x)$ is decreasing in $x$. Thus, by the IGFR property of $X$, we have $g_{H}(x)=x r_{H}(x)=\frac{x r_{F}(x)}{1-\bar{\theta} \bar{F}^{k}(x)}$ is increasing in $x$, i.e., $Y$ has IGFR property. Moreover,

$$
\lim _{x \rightarrow \infty} g_{H}(x)=\lim _{x \rightarrow \infty} \frac{x r_{F}(x)}{1-\bar{\theta} \bar{F}^{k}(x)}=\lim _{x \rightarrow \infty} x r_{F}(x)=l .
$$

Thus, by IGFR property of $Y$, it follows that $E\left(Y^{n}\right)$ is finite if, and only if, $l>n$ (see Theorem 2 in Lariviere, 2006).

In the following, we provide certain bounds for sf of a Harris family distribution using concepts of increasing (decreasing) failure rate (IFR) (DFR) and increasing 
(decreasing) average failure rate (IFRA) (DFRA) properties. In reliability, these aging properties are applied to examine how a component or a system improves or deteriorates with age. A non-negative $\mathrm{rv} X$ with hazard rate $r_{F}($.$) has IFR (DFR)$ property, if $r_{F}(x)$ is increasing (decreasing) in $\mathrm{x}$ and has IFRA (DFRA) property if $(-1 / x) \log \bar{F}(x)$ is increasing (decreasing) in $\mathrm{x}$.

Theorem 4.2. Let $X$ and $Y$ be two non-negative rv's corresponding to $F($.$) and$ $H(. ; \theta, k)$ in Eq. (1.3), respectively. Suppose that $X$ has IFR property, $\mu_{s}=\int_{0}^{\infty} t^{s} d F(t)$ and $\lambda_{s}=\mu_{s} / \Gamma(s+1), s \geq 1$. Then, we have

$$
\bar{H}(x ; \theta, k) \geq \begin{cases}\left(\frac{\theta}{\exp \left(\frac{x k}{\lambda_{s}^{1 / s}}\right)-\bar{\theta}}\right)^{\frac{1}{k}}, & x<\mu_{s}^{1 / s}, \\ 0, & x \geq \mu_{s}^{1 / s},\end{cases}
$$

Proof: By Eq. (1.3), $\bar{H}(x ; \theta, k)=\left(\frac{\theta}{\bar{F}^{-k}(x)-\bar{\theta}}\right)^{\frac{1}{k}}$. Since $X$ has IFR property, by Corollary 6.3 in Barlow and Proschan (1975), we have $\bar{F}^{-k}(x) \leq \exp \left(\frac{x k}{\lambda_{s}^{1 / s}}\right)$, for all $x<\mu_{s}^{1 / s}$. Thus, the assertion follows.

Theorem 4.3. Let $X$ and $Y$ be two non-negative rv's corresponding to $F($.$) and$ $H(. ; \theta, k)$ in Eq. (1.3), respectively. Suppose that $X$ has IFRA (DFRA) property with $p$-th quantile $\xi_{p}$. If $\theta \geq 1(0<\theta \leq 1)$, then,

$$
\bar{H}(x ; \theta, k) \begin{cases}\geq(\leq) \exp (-\alpha x), & x \leq \xi_{p}, \\ \leq(\geq) \exp (-\alpha x), & x \geq \xi_{p},\end{cases}
$$

where $\alpha=-\frac{1}{\xi_{p} k} \log \left(\frac{\theta q^{k}}{1-\bar{\theta} q^{k}}\right), q=1-p$.

Proof: If $X$ has IFRA (DFRA) property with p-th quantile $\xi_{p}$, then by Theorem 6.1 in Barlow and Proschan (1975)

$$
\bar{F}(x) \begin{cases}\geq(\leq) \exp (-\alpha x), & x \leq \xi_{p}, \\ \leq(\geq) \exp (-\alpha x), & x \geq \xi_{p},\end{cases}
$$

where $\alpha=\log \left(\bar{F}\left(\xi_{p}\right)\right)^{-1 / \xi_{p}}, q=1-p$. Since, if $\theta \geq 1(0<\theta \leq 1)$ and $X$ has IFRA (DFRA) property, then Y also has IFRA (DFRA) property (cf. Theorem 8 in Abbasi and Alamatsaz, 2015+) and since $\bar{H}\left(\xi_{p} ; \theta, k\right)=\left(\frac{\theta q^{k}}{1-\bar{\theta} q^{k}}\right)^{\frac{1}{k}}$, we have the results.

\section{Acknowledgements}

The authors are grateful to the Editor and two anonymous reviewers whose valuable suggestions lead to an improved version of the manuscript.

\section{References}

S. Abbasi and M. H. Alamatsaz. Preservation properties of stochastic orderings by transformation to Harris family. Preprint $(2015+)$.

R. A. Al-Jarallah, M. E. Ghitany and Ramesh C. Gupta. A proportional hazard Marshall-Olkin extended family of distributions and its application to Gompertz distribution. Comm. Statist. Theory Methods 43 (20), 4428-4443 (2014). MR3269284. 
M. H. Alamatsaz and S. Abbasi. Ordering comparison of negative binomial random variables with their mixtures. Statist. Probab. Lett. 78 (14), 2234-2239 (2008). MR2458032.

E.-E. A. A. Aly and L. Benkherouf. A new family of distributions based on probability generating functions. Sankhya B 73 (1), 70-80 (2011). MR2826321.

R. E. Barlow and F. Proschan. Statistical theory of reliability and life testing. Holt, Rinehart and Winston, Inc., New York-Montreal, Que.-London (1975). MR0438625.

A. Batsidis and A. J. Lemonte. On the Harris extended family of distributions. Statistics 49 (6), 1400-1421 (2015). MR3415226.

F. Belzunce, J. M. Ruiz and M. Carmen Ruiz. On preservation of some shifted and proportional orders by systems. Statist. Probab. Lett. 60 (2), 141-154 (2002). MR1945437.

M. Brown and J. G. Shanthikumar. Comparing the variability of random variables and point processes. Probab. Engrg. Inform. Sci. 12 (4), 425-444 (1998). MR1643827.

S. Eryilmaz and G. Y. Tutuncu. Relative behavior of a coherent system with respect to another coherent system. Statist. Papers 56 (2), 519-529 (2015). MR3331875.

M. E. Ghitany and S. Kotz. Reliability properties of extended linear failure-rate distributions. Probab. Engrg. Inform. Sci. 21 (3), 441-450 (2007). MR2348068.

W. Gui. Marshall-Olkin extended log-logistic distribution and its application in minification processes. Appl. Math. Sci. (Ruse) 7 (77-80), 3947-3961 (2013). MR3086017.

T. E. Harris. Branching processes. Ann. Math. Statistics 19, 474-494 (1948). MR0027465.

Y. Huang and G. Da. Some new stochastic comparison results on proportional odds models. Chinese J. Appl. Probab. Statist. 28 (1), 43-50 (2012). MR2951691.

J. Jarrahiferiz, G. R. Mohtashami Borzadaran and A. H. Rezaei Roknabadi. Some properties and applications of shifted proportional stochastic orders. Istatistik 6 (2), 80-91 (2013). MR3155181.

M. A. Jazi and M. H. Alamatsaz. Ordering comparison of logarithmic series random variables with their mixtures. Comm. Statist. Theory Methods 39 (18), 32523263 (2010). MR2747582.

M. A. Jazi, M. H. Alamatsaz and S. Abbasi. A unified approach to ordering comparison of GPS distributions with their mixtures. Comm. Statist. Theory Methods 40 (14), 2591-2604 (2011). MR2863332.

M. A. Lariviere. A note on probability distributions with increasing generalized failure rates. Oper. Res. 54 (3), 602-604 (2006). MR2232983.

M. A. Lariviere and E. L. Porteus. Selling to a newsvendor: An analysis of priceonly contracts. Manufacturing $\&$ Service Operations Management 3 (4), 293-305 (2001). DOI: 10.1287/msom.3.4.293.9971.

R. E. Lillo, A. K. Nanda and M. Shaked. Preservation of some likelihood ratio stochastic orders by order statistics. Statist. Probab. Lett. 51 (2), 111-119 (2001). MR1808033.

A. W. Marshall and I. Olkin. A new method for adding a parameter to a family of distributions with application to the exponential and Weibull families. Biometrika 84 (3), 641-652 (1997). MR1603936. 
T. Nakai. A partially observable decision problem under a shifted likelihood ratio ordering. Math. Comput. Modelling 22 (10-12), 237-246 (1995). Stochastic models in engineering, technology and management (Gold Coast, 1994).

A. K. Nanda and S. Das. Stochastic orders of the Marshall-Olkin extended distribution. Statist. Probab. Lett. 82 (2), 295-302 (2012). MR2875214.

H. M. Ramos Romero and M. A. Sordo Díaz. The proportional likelihood ratio order and applications. Qüestiió (2) 25 (2), 211-223 (2001). MR1904970.

M. Shaked and J. G. Shanthikumar. Stochastic Orders. Springer Series in Statistics. Springer-Verlag New York (2007). ISBN 978-0-387-32915-4. DOI: 10.1007/9780-387-34675-5.

S. K. Singh and G. S. Maddala. A function for size distribution of incomes. Econometrica 44, 963-970 (1976).

N. Torrado. Comparisons of smallest order statistics from Weibull distributions with different scale and shape parameters. J. Korean Statist. Soc. 44 (1), 68-76 (2015). MR3304006.

Y. Zhou, Z. Zhang, T. R. Lin and L. Ma. Maintenance optimisation of a multi-state seriesparallel system considering economic dependence and state-dependent inspection intervals. Reliability Engineering $\mathscr{E} 3$ System Safety 111, 248-259 (2013). DOI: $10.1016 /$ j.ress.2012.10.006. 